\title{
Clues to the formation of the Milky Way's thick disk
}

\author{
M. Haywood ${ }^{1}$, P. Di Matteo ${ }^{1}$, O. Snaith ${ }^{1, \star}$, and M. D. Lehnert ${ }^{2}$ \\ ${ }^{1}$ GEPI, Observatoire de Paris, CNRS, Université Paris Diderot, 5 place Jules Janssen, 92190 Meudon, France \\ e-mail: Misha.Haywood@obspm.fr \\ 2 Institut d'Astrophysique de Paris, CNRS UMR 7095, Université Pierre et Marie Curie, 98bis Bd Arago, 75014 Paris, France
}

Received 4 December 2014 / Accepted 25 March 2015

\section{ABSTRACT}

\begin{abstract}
We analyze the chemical properties of a set of solar vicinity stars, and show that the small dispersion in abundances of $\alpha$-elements at all ages provides evidence that the star formation history (SFH) has been uniform throughout the thick disk. In the context of longtimescale infall models, we suggest that this result points either to a limited dependence of the gas accretion on the Galactic radius in the inner disk $(R<10 \mathrm{kpc})$ or to a decoupling of the accretion history and SFH due to other processes governing the interstellar medium in the early disk, suggesting that infall cannot be a determining parameter of the chemical evolution at these epochs. We argue that these results and other recent observational constraints - namely the lack of radial metallicity gradient and the non-evolving scale length of the thick disk - are better explained if the early disk is viewed as a pre-assembled gaseous system, with most of the gas settled before significant star formation took place - formally the equivalent of a closed-box model. In either case, these results point to a weak or non-existent inside-out formation history in the thick disk or in the first 3-5 Gyr of the formation of the Galaxy. We argue, however, that the growing importance of an external disk whose chemical properties are distinct from those of the inner disk would give the impression of an inside-out growth process when seen through snapshots at different epochs. The progressive, continuous process usually invoked may not have actually existed in the Milky Way.
\end{abstract}

Key words. Galaxy: abundances - Galaxy: evolution - Galaxy: disk - Galaxy: formation

\section{Introduction}

The cosmic star formation history (SFH) shows that galaxies went through their most vigorous phase of star formation at redshift $z=1.5-3$ (e.g., Madau \& Dickinson 2014). Structures thought to have formed at this epoch in disk galaxies are classical bulges and thick disks. The stellar content of the nearby universe in environments with average density is dominated by pure disk galaxies (Kormendy et al. 2010; Fisher \& Drory 2010; Laurikainen et al. 2014). This suggests that classical bulges represent only a minor mass component of galaxies. Thick disks, however, are found to be ubiquitous, and are detected on nearly all nearby edge-on disks galaxies (Comerón et al. 2011). Recent results (Ness et al. 2013a,b; Di Matteo et al. 2014, 2015) have confirmed the view that the Milky Way (MW) is also a pure disk galaxy (Shen et al. 2010; Kunder et al. 2012), i.e., it has no substantial classical bulge. Furthermore, measurements of its SFH have shown that during the peak of star formation in the universe, the MW was a star-bursting galaxy and was forming its thick disk (Snaith et al. 2014, 2015; Lehnert et al. 2014). The structural parameters of this population, with a short scale length (Bensby et al. 2011; Cheng et al. 2012a; Bovy et al. 2012) suggest that it constitutes most of the mass in the MW's central region (Ness et al. 2013a,b; Di Matteo et al. 2014, 2015; Haywood et al., in prep.).

This emerging scheme suggests that the formation of the thick disk is a crucial phase in the formation of disks (see Lehnert et al. 2014) because this population could represent a

* Current address: Department of Physics \& Astronomy, University of Alabama, Tuscaloosa, Albama, USA. substantial fraction of the mass of galaxies and because it may account for the main increase in the metal enrichment of galaxies since the formation of the first stars. These views are opposite to the standard chemical evolution scenario based on long-term infall (e.g., Chiappini et al. 1997; Fraternali 2014) and the recognition that the solar vicinity contains too few low-metallicity dwarf stars compared to the "closed-box" model. In other words, observations of the solar vicinity would suggest that the enrichment rate has been faster than the star formation rate (SFR). Infall models have been introduced in order to solve this problem by limiting the dilution of ejected metals to a disk parsimoniously provisioned with gas, and by boosting the increase of the metallicity at early times without creating too many metal-poor stars. Hence, these scenarios leave no room for a substantial component of intermediate metallicity in the MW. This credo has been questioned by a new scheme presented in Haywood et al. (2013), Snaith et al. (2014, 2015), and Haywood (2014a,b).

Another important feature of the standard chemical evolution scenario is to make the accretion of gas radially dependent, with slower accretion outward, in order to mimic an inside-out formation. Inner regions form their stars first, because gas density increases faster, while outer regions grow at later times and at a slower pace. Because of the coupling assumed between accretion and SFR in these models, a natural signature of such a scheme would therefore be that SFHs vary with distance from the Galactic center. Direct measurement of the star formation history outside the solar vicinity is not feasible currently; however, we know that a given SFH leaves specific signatures on the radial and temporal dependence of the chemical abundances in stars (Haywood et al. 2013; Snaith et al. 2014, 2015). Since thick disk stars observed in the solar vicinity were born over a 
wide range of galactocentric distances (see Sect. 2), their chemical properties should therefore reflect the variety in the radial SFHs of the thick disk, if any.

In the following, we show that these chemical properties are in fact remarkably homogeneous at all ages in the thick disk, leaving little room for significant variation in the SFH of this population as a function of radius. The outline of the paper is as follows: in the following section we briefly describe our data, in Sect. 3 we describe the model and present our results, and we discuss these results in Sect. 4.

\section{Data}

We use the sample of Adibekyan et al. (2012) already analyzed in Haywood et al. (2013). The sample contains 363 stars with age determinations, to which we added the 36 stars from the sample of Nissen \& Schuster (2010) for which we could determine ages. Ages for this last sample were determined in the same manner as those from the Adibekyan et al. (2012) sample in Haywood et al. (2013). However, as reported by Schuster et al. (2012), the effective temperature of the stars in Nissen \& Schuster may have been underestimated. We checked this statement by comparing the 11 HIPPARCos stars in common between the two samples. All these objects have a lower temperature in Nissen \& Schuster (2010) than in Adibekyan et al. (2012), with a mean offset of $-75 \mathrm{~K}$, which confirms the statement of Schuster et al. (2012). Following their recommendation, we added $+100 \mathrm{~K}$ to the Nissen \& Schuster (2010) values.

In Haywood et al. (2013) the spectroscopic scale of Adibekyan et al. (2012) was compared to photometric temperatures using the $T_{\text {eff }}-V-K$ calibration of Casagrande et al. (2010), from which we deduced that the two scales are off by $56 \mathrm{~K}$. The impact of this offset on stellar ages of such differences in temperatures was studied in Haywood et al. (2013) and found to be limited (see their Fig. 2). Ages were determined using the Bayesian method of Jørgensen \& Lindegren (2005), and implies the derivation of a probability distribution function (PDF) for each star. We adopted the Yonsei-Yale set of isochrones, with atmospheric parameters taken from the study of Adibekyan et al. (2012), including mean $[\alpha / \mathrm{Fe}]$ values. The uncertainties on the ages are derived from the PDF as described in Jørgensen \& Lindegren (2005), and the values (see Fig. 3) are for a confidence level of $68 \%$, corresponding to $\pm 1 \sigma$ Gaussian errors. Random uncertainties on age reflect the uncertainties on the atmospheric parameters. In Haywood et al. (2013), we assumed $50 \mathrm{~K}$ uncertainty on effective temperatures, noting that the formal uncertainties quoted by Adibekyan et al. (2012) are sometimes much smaller than this, 0.1 dex on metallicities and $0.1 \mathrm{mag}$ on absolute magnitudes. The total uncertainties in the ages, as described in Haywood et al. (2013), are on the order of $1 \mathrm{Gyr}$ for the random error (with a mean uncertainty of $1.1 \mathrm{Gyr}$ ), and with a systematic error due to stellar physics of 1 to 1.5 Gyr. The systematic uncertainties were estimated by comparing the ages determined using different sets of isochrones (see Haywood et al. 2013, for details) Given the dispersion of points in Fig. 3, of the order of $1 \mathrm{Gyr}$, we deemed our error estimates on age as realistic.

The $[\alpha / \mathrm{Fe}]-[\mathrm{Fe} / \mathrm{H}]$ distribution of the whole sample is shown in Fig. 1. The stars below the separating line are considered either as metal-poor thin disk objects (see discussion on these objects in Haywood 2008 and Haywood et al. 2013 for the status of these objects, and Snaith et al. 2014, 2015 for a description of their chemical evolution), or as accreted stars, following the study by Nissen \& Schuster (2010).

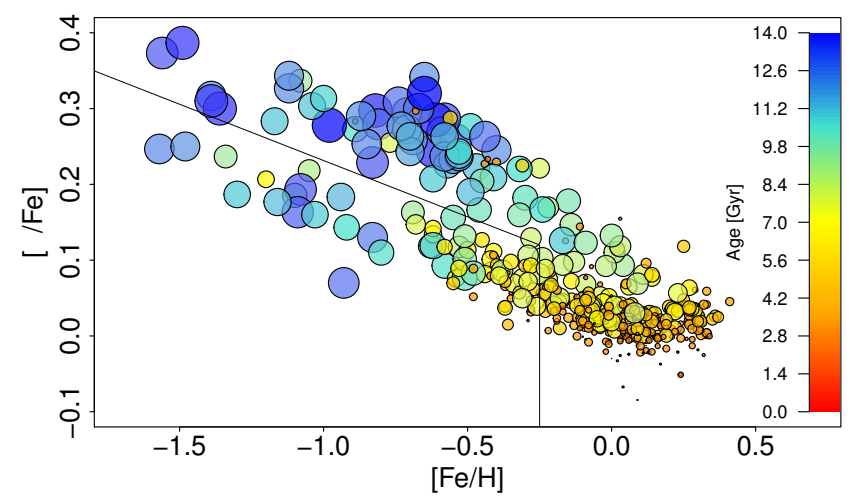

Fig. 1. $[\alpha / \mathrm{Fe}]-[\mathrm{Fe} / \mathrm{H}]$ distribution at the solar vicinity for our sample. The colors (indicated in the legend) and size (larger implies older) of the symbols indicate the age of the stars. The stars below and to the left of the line are either accreted stars according to Nissen \& Schuster (2010; for those objects that have $[\mathrm{Fe} / \mathrm{H}]<-0.7 \mathrm{dex}$ ), or metal-poor thin disk stars. They are represented by empty circles in Fig. 2, and removed from the age-alpha distribution in Fig. 3.

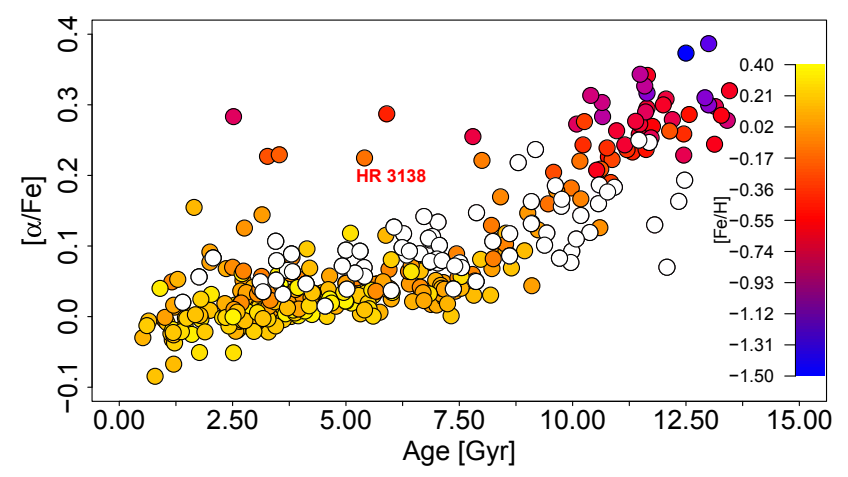

Fig. 2. Age- $[\alpha / \mathrm{Fe}]$ distribution of the stars in Fig. 1. Metal-poor thin disk stars and accreted stars, as selected from Fig. 1 (below and to the left of the limits in gray), are shown as white dots.

In Fig. 2, which shows the age- $[\alpha / \mathrm{Fe}]$ distribution (the color now coding the metallicity of each star), these objects are represented by empty circles. The final distribution of Age- $[\alpha / \mathrm{Fe}]$, after removing accreted and metal-poor thin disk stars, is plotted in Fig. 3. The sequences of the thin and thick disks appear clearly in the plot, with a sharp change of slope between the two at $\sim 8$ Gyr, as first discussed in Haywood et al. (2013).

A number of objects with $\alpha$-enhanced abundance ratios are more clearly demonstrated here than in Haywood et al. (2013); they are younger than the sequence of thick disk objects (age $<8-9$ Gyr at $[\alpha / \mathrm{Fe}] \sim 0.2-0.3 \mathrm{dex})$. As we already discussed in Haywood et al. (2013), these objects have no peculiarities. We did not note at that time, however, the dedicated study of Fuhrmann et al. (2012) on HIP 38908 (HR 3138). Fuhrmann et al. (2012) found a similar "young" age for this object: we derive an age of $5.4_{-2.3}^{+1.4} \mathrm{Gyr}$, while Fuhrmann et al. (2012) give $5.6_{-1.8}^{+2.2}$ Gyr. They commented further on the fact that radial velocity excludes the possibility that HR 3138 is a blue straggler with a white dwarf. The authors could not find any specific reason for the young age, but suggested that either the star had accreted gas from interstellar material or that the enhanced 


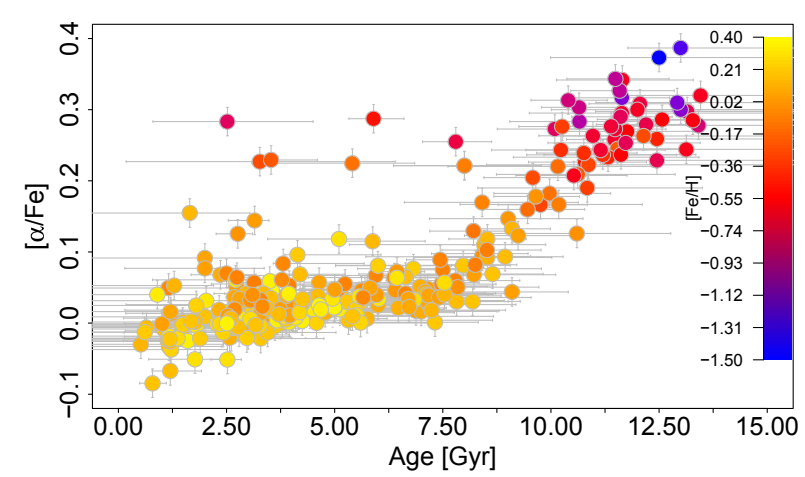

Fig. 3. Age- $[\alpha / \mathrm{Fe}]$ distribution at the solar vicinity after removing metal-poor thin disk stars and accreted stars, as selected from Fig. 1. We note the remarkably small dispersion in both the thin and thick disk sequences of stellar age and $[\alpha / \mathrm{Fe}]$. Errors on age determinations are described in the text. On $[\alpha / \mathrm{Fe}]$, the 0.02 dex error is taken as representative of the internal errors given in Adibekyan et al. (2012).

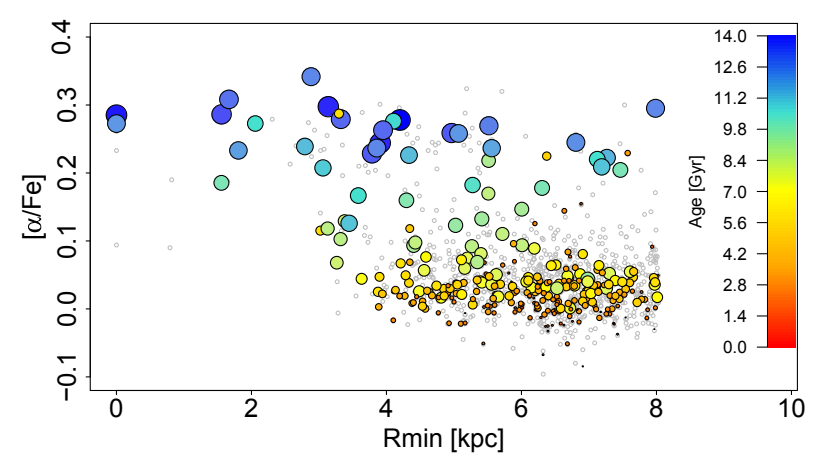

Fig. 4. Mean alpha abundance as a function of pericenter distance. Color (indicated in the legend) and size of the symbols indicate the age (larger symbols implies older ages) of the stars. Thick disk stars are essentially those stars with $[\alpha / \mathrm{Fe}]>0.1$ dex. Gray points are for the whole sample.

effective temperature and luminosity resulted from a merger with a companion ${ }^{1}$.

In Fig. 4 , we plot $[\alpha / \mathrm{Fe}]$ as a function of the pericenter distance of stars. The purpose of this plot is to illustrate that the pericenters of thick disk stars span the whole range of radii from $8 \mathrm{kpc}$ to the innermost regions. Hence, unless the orbital characteristics of these stars were seriously affected by dynamical evolution, it can be assumed that they represent a reasonable sampling of the thick disk over a wide range, perhaps even the whole range, of radial distances from the Galactic center.

\section{Results: Modeling the $[\alpha / \mathrm{Fe}]$-age relation}

\subsection{The model and chemical tracks}

We used a closed-box model, which is discussed at length in Snaith et al. $(2014,2015)$. We note that the effects described here are expected to be of similar or even higher amplitudes than with standard infall models. The reason being that since the interstellar medium (ISM) is less important in these models, they

\footnotetext{
1 The other stars in our sample with similar characteristics HIP 69780, 77637, 51191, 54469, 77610, 109822 - have no known peculiarity. Most (HIP 69780, HIP 77637, HIP 51191, HIP 54469 and HIP 109822) have clear asymmetric drift and overall hot kinematics, confirming that they should belong to the thick disk or halo, in spite of their age. These objects are not considered further in the present study.
}

are more sensitive to the level of metal injection in the ISM than closed-box models, which have greater inertia in this respect. A clear illustration of the effect of the variation of the SFHs on increasing the dispersion of alpha abundances at a given age is given in Minchev et al. (2013), their Fig. 7.

In the following, we use the nomenclature of the disks as given in Haywood et al. (2013). That is, we consider that the thick and thin disk up to radial distances of $10 \mathrm{kpc}$ form the inner disk, while stars beyond this limit are members of the outer disk. We sketched a scenario for the formation of these two different structures in Haywood et al. (2013) and the chemical evolutions of both are described in Snaith et al. (2015).

In short, the closed-box model assumes that (1) all the gas is in the system at the beginning. We take the closed-box model as the approximation of the case where most accretion in the inner disc $(R<10 \mathrm{kpc})$ occurred early; (2) the ISM is initially devoid of metals and remains well mixed during its whole evolution; and (3) no coupling between the SFR and the gas content is assumed. In Snaith et al. (2014, 2015), we determined the SFH by fitting our model to the Age-[Si/Fe] data. Here we assume different SFHs from which we derive the Age- $[\mathrm{Si} / \mathrm{Fe}]$ tracks which are compared to the data.

The ingredients of the model are standard theoretical yields (Iwamoto et al. 1999; Nomoto et al. 2006; Karakas 2010), an initial mass function (Kroupa 2001) independent of time, and a stellar mass-lifetime relation dependent on the metallicity (Raiteri et al. 1996). No instantaneous recycling approximation has been implemented, see Snaith et al. (2015) for details.

\subsection{Comparison with the data}

Models with radially dependent accretion timescales have a range of SFHs that vary from having a peak at early times in the inner disk to constant or even increasing SFH in the solar vicinity or beyond (e.g., Naab \& Ostriker 2006; Minchev et al. 2013; Kubryk et al. 2015). Hence, in Minchev et al. the SFR at $R=4 \mathrm{kpc}$ peaks at $\sim 30 M_{\odot} \mathrm{pc}^{-2} \mathrm{Gyr}^{-1}$ in the first Gyr, decreasing to less than $10 M_{\odot} \mathrm{pc}^{-2} \mathrm{Gyr}^{-1}$ at the present time, while it only slightly decreases (by a factor of $\sim 2$ ) at the solar radius, and is very nearly constant at $12 \mathrm{kpc}$. In Naab \& Ostriker, the SFH at $4 \mathrm{kpc}$ peaks a later time (6 Gyr), reaching similar values $\left(20-30 M_{\odot} \mathrm{pc}^{-2} \mathrm{Gyr}^{-1}\right)$, and decreases to about $10 M_{\odot} \mathrm{pc}^{-2} \mathrm{Gyr}^{-1}$ at the present epoch. The peak of the SFR at the solar vicinity is reached only at $\sim 11 \mathrm{Gyr}$ and thus mostly increases throughout the evolution of the MW. Independently of any particular SFH, these models show that the SFR at early times varies by large amounts depending on the radius; there is a factor of about 5 to 10 at $2-4$ Gyr between the SFR in the inner regions $(2-4 \mathrm{kpc})$ and the outer regions (at about 8-10 kpc from the Galactic center).

Because the radial variation of the SFHs can vary widely between one model and another, we do not rely on any particular model. We have chosen a set of SFHs that we take as representative of the general phenomenology of radially dependent SFHs in disks, and that are shown in Fig. 5. They are taken as illustrating the range of variation that one can expect from adopting an infall timescale and related SFH varying with Galactic radius - increasing SFH, constant SFH, decreasing SFH - and reflect the 5-10 amplitude of the SFR in the first few Gyr as noted above.

Figure 6 shows the corresponding chemical tracks generated from these SFHs over-plotted on the data. As can be seen, the range of alpha abundances spanned by the models at any given age is large, bracketing the observed $[\mathrm{Si} / \mathrm{Fe}]$ values. As expected, 


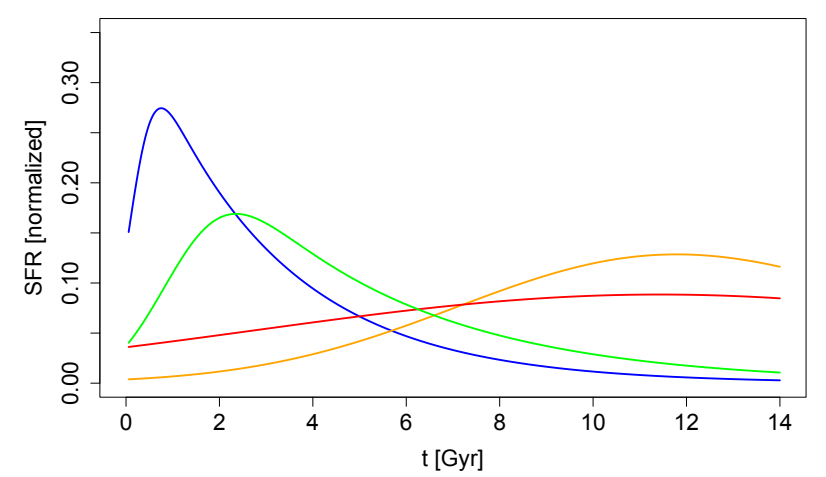

Fig. 5. Set of SFHs used in this analysis.

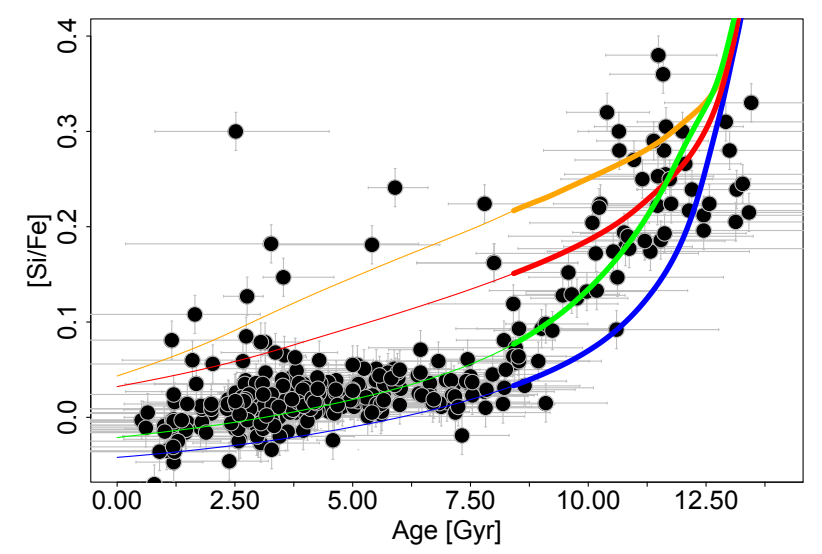

Fig. 6. Age vs. $[\mathrm{Si} / \mathrm{Fe}]$ chemical evolution tracks corresponding to the SFHs given in Fig. 5, compared to the stellar age vs. [Si/Fe] data. The colors of each line correspond to those in Fig. 5. The thick part of the curves correspond to the thick disk phase.

the SFH that fits best the observed age- $[\mathrm{Si} / \mathrm{Fe}]$ distribution is one with a significant peak of star formation at early times, similar to the one deduced by fitting the data in Snaith et al. (2014). If the peak in the SFH occurs too early $(<2 \mathrm{Gyr})$, the model generates too much iron early on and the chemical track is too low compared to the data. However, if the SFH is flat (constant SFR), the knee at $\sim 8$ Gyr is not reproduced, and the chemical track deviates from the data by about 0.1 dex over this region. In the case where the SFR starts low and increases to reach a maximum in the last few Gyrs as suggested by the model of Naab \& Ostriker (2006) for the solar vicinity, the model is very different from the data.

As shown in the previous section, the pericenter distances of thick disk stars span a wide radial range from less than $2 \mathrm{kpc}$ from the Galactic center up to the solar circle (Fig. 4). Hence, we expect thick disk stars to sample the whole thick $\operatorname{disk}(R<10 \mathrm{kpc})$ and their alpha abundance ratio to reflect SFHs at a variety of radii. Obviously, the expected dispersion is not observed. The thick and thin disk sequences are very well defined and the dispersion in abundance ratio is small at all ages. In fact, the observed dispersion at a given $[\mathrm{Si} / \mathrm{Fe}]$ is fully compatible with the uncertainty of about $1 \mathrm{Gyr}$ on the derived ages (Haywood et al. 2013), suggesting the intrinsic dispersion in abundance is essentially negligible. In particular, we would expect the transition from the thick to the thin disk at $\sim 8 \mathrm{Gyr}$ to be much less defined than it is. Had the thick disk SFHs been different in the inner and outer regions, the alpha abundance ratios at the end of the thick disk phase would show a level of dispersion in their abundance ratios which is not observed. As commented in Haywood et al. (2013), the data strongly suggest that the thick disk phase ended by leaving a disk of uniform properties from which the thin disk started to form.

While the previous discussion was meant to be illustrative and useful for comparing with the SFH commonly used in models, the most pertinent question is, How much variation in the SFH is really allowed by the data? This can be estimated by using the bootstrap SFHs generated in Snaith et al. (2015) to evaluate the uncertainty in the mean SFH that fits the data. The bootstrap SFHs give us some kind of "cosmic variation" that is allowed by the constraints provided by the data. What we seek here is not the uncertainty in the SFR at a given time, but how much the SFH can be systematically below or above the best fit SFH over a given time interval. In order to estimate this, we summarize the information given by each of the bootstrapped SFHs by measuring the ratio of the stellar mass formed during the thick disk phase (13-8 Gyr) to the total stellar mass formed (13-0 Gyr), for each solution. The mean and dispersion of these percentages are 56 and $2.5 \%$, with values that vary, at most, between 50 and $61 \%$. We note that the derived uncertainty in the SFR at a given age is much larger than $2.5 \%$ (see the error bars in the derived SFH in Snaith et al. 2014, 2015), but the overall variation of the SFR, as measured by the percentage of mass created in the age range 13-8 Gyr relative to the total stellar mass, is much better constrained. Comparatively, the stellar mass produced between ages 13 and $8 \mathrm{Gyr}$ - during the thick disk phase - relative to the total stellar mass in each of the model SFHs (Fig. 5) is 11, 26, 33, and 63\%. Hence, any significant variations from the mean SFH derived in Snaith et al. (2014, 2015) are ruled out.

\section{Discussion}

Summarizing the previous section, the observed dispersion in the age- $[\mathrm{Si} / \mathrm{Fe}]$ distribution essentially reflects the uncertainties in the derived ages, although the thick disk stars originate from all over the disk. This implies that the chemical evolution in the thick disk phase must have been an extremely homogeneous process, incompatible with significant spatial variations of the SFR at these epochs. This is in clear contradiction with a number of models, which explicitly predict a very significant dispersion, see for example Minchev et al. (2013), or Kubryk et al. (2015), which both have a range of $[\alpha / \mathrm{Fe}]$ larger than 0.2 dex at the end of the thick disk phase. We note, however, that this is likely to be true for all models which, based on the inside-out scenario, build their disks through a radially dependent SFH.

\subsection{Radially uniform SFH of the thick disk}

There are a number of important consequences of this result. In chemical evolution models based on long-timescale accretion of gas (e.g., Chiappini et al. 1997; Minchev et al. 2013; Kubryk et al. 2015), the SFH depends on the infall history through some form of relation between the star-formation intensity and the gassurface density (i.e., a Schmidt-Kennicutt law). The infall law is chosen in order to reproduce the phenomenology of insideout galaxy formation models and one of their possible signatures, chemical radial gradients. Our results contradict a fundamental aspect of these scenarios: since the SFH is measured to be the same across the (thick) disk, it may likely imply that there is no radial dependence of the accretion of gas onto the inner disk $(R<10 \mathrm{kpc})$. Although such simplistic inferences are 
similar to those assuming direct connection between infall and star formation intensity, they should be interpreted with caution because as shown recently (Hopkins et al. 2014; Woods et al. 2014), the SFH does not necessarily correlate with the accretion history. Feedback is expected to play an important role by reducing the SFR and efficiency thereby producing a reservoir of gas that can affect the long-term fueling of the SFH of a galaxy. Hence, infall could be radially dependent, but not directly correlated to the SFR, implying that it cannot be a determining parameter of the chemical evolution in the inner disk.

Another perhaps more natural explanation could explain the seemingly uniform SFH in the thick disk. In Haywood (2014a,b) and Snaith et al. (2014, 2015), we provided a number of arguments to propose a new scenario for the chemical evolution of the MW disk. We argued that the structural parameters and the measurement of the SFH of the MW provide new evidence that the thick disk is massive, and that this is not expected in standard chemical scenarios (see the Introduction). The only possible way to generate sufficient numbers of stars at intermediate metallicities (which, we argued, are mostly confined to the inner regions, as one would expect for a population with $2 \mathrm{kpc}$ scale length; Bensby et al. 2011; Cheng et al. 2012a; Bovy et al. 2012), is that the gaseous disk was already massive at early times. From the point of view of chemical evolution, the model that is the most likely to represent this situation is a closed-box model. In this case, because of the short scale length of the disk, the relative large scale of the turbulence (Lehnert et al. 2014), and the absence of a radially dependent accretion timescale, less differentiation in the SFHs is expected to take place in the inner and outer regions of the thick disk.

Nidever et al. (2014) confirm this conclusion on the basis of the analysis of the APOGEE data and a chemical evolution model. Their model includes infall with a long timescale but no radial dependence, and so there is no induced variation in the SFH due to some kind of inside-out formation. However, they assessed possible variations in the SFH by assuming different star formation efficiency, and deduced that some homogeneity in the SFH can be deduced from the limited dispersion observed in the $[\alpha / \mathrm{Fe}]-[\mathrm{Fe} / \mathrm{H}]$ distribution in the thick disk regime sampled by the APOGEE data. As in Haywood et al. (2013) and Lehnert et al. (2014), they note that this implies that the thick disk formed from a turbulent, well-mixed gaseous disk.

\subsection{Inside-out galaxy formation model}

Other evidence supports a uniform SFH for the thick disk. First, no radial metallicity gradients have been found in the thick disk (Cheng et al. 2012b). This is expected if the SFH is radially undifferentiated. We note that the tight correlation found between age and chemical parameters (see Haywood et al. 2013, and previous section) in the thick disk implies that the lack of gradient is not a consequence of mixing in the stellar disk, but of a well mixed ISM at a given epoch in the thick disk (Haywood et al. 2013). The chemical properties of the thick disk evolved homogeneously over time. We already commented in Haywood et al. (2013) and Lehnert et al. (2014) that this was most probably a consequence of the high turbulence in the gaseous disk at these epochs. Turbulence induces sufficient mixing to explain the high homogeneity of chemical species in the ISM (Feng \& Krumholz 2014). Stars formed out of this well-mixed gas in a geometrically thick layer.

Second, no dependence of the radial scale length of the thick disk as a function of the $\alpha$-abundance ratios has been detected (see our comments in Haywood et al. 2013 in the Fig. 5 of Bovy et al. 2012). Haywood et al. (2013) demonstrated that alpha abundances correlate well with age in the thick disk phase (see also the previous section). Hence, this implies that no significant dependence of the scale length with age, or an inside-out age trend during the formation of the thick disk, is detected. This is in accordance with the fact that no significant variation of the SFH is imprinted in the age- $\alpha$ relation, which we would expect if the youngest populations had the longest scale lengths. This is also in good agreement with the general scheme presented in Haywood et al. (2013) where we discussed the inside-out galaxy formation scenario.

We note that the Haywood et al. study has been cited as claiming evidence that the MW did not form inside-out. We caution that this is an oversimplification of the view presented in that paper. What we claimed is that there is no such structure as a single disk formed smoothly and continuously from the inside-out and generating smooth radial chemical gradients. Indeed, there are two disks in our nomenclature (see Haywood et al. 2013): the inner disk $(R<9-10 \mathrm{kpc})$, composed of a thick and a thin disk, with different scale lengths ${ }^{2}$ ( $\sim 2$ and $3.6 \mathrm{kpc}$, see Bovy et al. 2012), but with no evidence of an increase in the scale length as each of these disks grew, and the outer disk, which started to form before the inner thin disk, $10 \mathrm{Gyr}$ ago, and which has a long scale length ( $\sim 4 \mathrm{kpc}$ or more). Hence, the general trend at early times ( $>8 \mathrm{Gyr}$ ) would be mostly imprinted by the formation of the massive and concentrated thick disk, followed by the outer (but less dense) disk which has a much longer scale length. Hence, snapshots of such a galaxy at different epochs would certainly give the impression of an inside-out growth of its disk, although the overall sequence is more complex and is not a smooth process. What we did claim in Haywood et al. and now argue further is that this overall scenario is in contradiction with the simplest picture of inside-out galaxy formation as we now discuss.

\subsection{The formation of disks}

Within the context of these results, models of formation of MW-like galaxies may need some, perhaps significant, revisions. Models where the radial dependent gas accretion drives a sequence of star formation that is inside-out during the formation of the thick disk are now likely ruled out. In addition, models, at least during the phase of thick disk growth, need to be such that the exponential disk scale length does not increase. Ferguson \& Clarke (2001) explored how the scale length of disks is affected during infall when the timescales of star formation and viscous gas redistribution are similar (within a factor of a few). They show that, if the main infall phase precedes the onset of star formation (the equivalent of a closed-box model), the scale length is invariant with time, because the scale length is determined by the mean specific angular momentum of the gas already settled and viscosity redistributes the angular momentum such that the scale length is invariant even as the total extent of the disk grows. On the contrary, if the infall timescale is similar to the lifetime of the disk, the scale length evolves according to the specific angular momentum of the accreted gas. Hence,

2 The value of $3.6 \mathrm{kpc}$ is a representative mean, but at $[\mathrm{Fe} / \mathrm{H}]>$ -0.1 dex the scale length measured by Bovy et al. (2012) for the thin disk is a complex function of metallicity and alpha abundance. For instance, metal-rich stars, which are more likely to belong to the inner thin disk have a scale length significantly shorter than the value of $3.6 \mathrm{kpc}$. This complex issue is discussed in Haywood et al. (in prep.). 
at first order, a non-evolving scale length in the thick disk is a natural outcome of a pre-existing disk rich in gas. Interestingly, Ferguson \& Clarke predict that in their pre-assembled disk "half of the stellar mass was in place more than 6 Gyr ago", in good agreement with the SFH measured by Snaith et al. (2014, 2015).

The advantage of viscous dissipation is that it naturally forms exponential disks with a wide span in radius (Ferguson \& Clarke 2001) and because the viscous timescale is similar to that of the star formation timescale ${ }^{3}$, it helps to alleviate the problem of forming massive bulges which plague simulations without strong stellar feedback (Dutton \& van den Bosch 2009). Viscosity both moves gas outward, extending the disk, and prevents gas from accumulating into the center of the potential. Since the disk scale length does not increase when both viscosity is important and the accretion and star formation timescales are similar, gas is distributed with the same radial dependence as the previous generation of stars. Thus viscosity is able to prevent the thick disk from developing a radial metallicity gradient or from increasing its scale length with time or with increasing metallicity.

We note that the analysis of Vollmer \& Leroy (2011) suggests that star formation in local disk galaxies with stellar mass $M_{\star} \lesssim 10^{10} M_{\odot}$ may also be sustained by the viscous dissipation of the (outer) HI extended disks. Viscous dissipation allows the gas to move inward and distributes it radially within the disk at rates sufficient to support the observed rate and distribution of star formation. Their analysis of local disk galaxies and our results imply that viscous dissipation and gas redistribution may be generically important to the growth of disks.

Gas accretion, regulated by the intensity of ongoing star formation, may help to alleviate both the problem of needing the gas accretion to follow the radial distribution of the star formation and of maintaining a constant disk scale length. For example, Marinacci et al. (2011) and Marasco et al. (2012) have suggested gas driven into the hot coronae by recent star formation in the disk may cause the halo gas to cool. This cool gas can then accrete onto the galaxy. Since the rate at which mass and momentum are injected into the halo gas controls how much gas is ultimately accreted, and these injection rates depend on the star formation intensity of the disk, a self-regulating cycle is generated whereby more intense star formation leads to more intense accretion, resulting in further star formation, and so on.

In such a model, unlike the recent models of gas accretion from the cosmic web (e.g., Danovich et al. 2015), the gas accretion rate would follow the radial intensity of on-going disk star formation and, perhaps in conjunction with viscosity, maintain a constant disk scale length.

The results of the study by van Dokkum et al. (2013) fits well in this scheme, first because of a similar mass growth found on progenitors of the MW (see Snaith et al. 2014; Lehnert et al. 2014), but also by showing that their galaxies do not increase their size through inside-out growth between $z=2.5$ and 1 , but

\footnotetext{
3 The viscosity is given by $v=v_{\text {turb }} l_{\text {turb }}$, where $v_{\text {turb }}$ is the turbulent velocity of the largest energy injection scale and $l_{\text {turb }}$ is this largest scale. The viscous timescale is simply $t_{v}=R^{2} / v$ (Vollmer \& Leroy 2011). Scaling this relation to observations of $z=2-3$ galaxies and values consistent with the thick disk of the MW (Bovy et al. 2012; Haywood et al. 2013; Lehnert et al. 2014), suggests $v=0.05\left(v_{\text {turb }} / 50 \mathrm{~km} \mathrm{~s}^{-1}\right)$ $\left(l_{\text {turb }} / 1000 \mathrm{pc}\right) \mathrm{pc}^{2} \mathrm{yr}^{-1}$ and $t_{v}=6 \times 10^{7}(R / 1.8 \mathrm{kpc})^{2} \mathrm{yr}$. The global rate at which stars form is approximately one dynamical time of the thick disk, $t_{\text {dyn }}=6 \times 10^{7}(R / 1.8 \mathrm{kpc})\left(v_{\text {rot }} / 200 \mathrm{~km} \mathrm{~s}^{-1}\right)^{2} \mathrm{yr}$. These crude estimates suggest that plausibly $t_{v} \approx t_{\text {dyn }}$ or similar to the star formation timescale of the disk, and thus viscosity may be important for redistributing the gas.
}

through self-similar mass growth at all radii (see also Morishita et al. 2015). Similarly, Nelson et al. (2012) find that galaxies begin to form inside-out after $z=1$ (see comment by van Dokkum et al. 2013). This is precisely what is predicted by viscous redistribution of the gas (Ferguson \& Clarke 2001), and in agreement with our findings here.

\section{Conclusions}

We have studied the impact of varying the SFH on the age$[\alpha / \mathrm{Fe}]$ relation of disk stars. We show that a radially dependent growth of the disk would give rise, through a radial variation of the SFHs, to a dispersion in this relation which is not observed. Star formation in the thick disk was a general, large scale process that led to a homogeneous chemical evolution throughout the inner disk $(R<10 \mathrm{kpc})$. Consequences of these results for chemical evolution models have been explored. If infall intervenes on a long timescale, as advocated in standard chemical evolution models, it implies either that infall does not vary with Galactocentric distance in the inner disk, or that infall is decoupled from the SFH. In either case, it implies that infall is not a determining parameter of the chemical evolution of the inner disk. In Haywood (2014a,b), Snaith et al. (2014, 2015), we have shown that the characteristics of the thick disk favor a scenario where stars started to form from a disk rich in gas (most accretion occurred before significant star formation took place), which we model as a closed box. We argued that the present results find better justification within this new context. It is reasonable to expect that a uniform SFH is more likely to occur in a disk with a short scale length and given the high level of turbulence in disks at high redshift, and with no radially dependent infall timescale. Moreover, differentiated SFHs are expected to produce both radial chemical gradients and an increase in the scale lengths of the disk, expected in inside-out formation scenarios, while none are seen in the observations. The study by Ferguson \& Clarke (2001) is qualitatively in agreement with our results; they argue that, under the assumption of similar timescales for star formation and viscosity in a pre-assembled disk, no significant variation of the scale length is expected, and therefore no inside-out growth of the disk.

Acknowledgements. The authors gratefully acknowledge the referee for helpful and constructive comments on the paper. This research has made use of the SIMBAD database, operated at CDS, Strasbourg, France.

\section{References}

Adibekyan, V. Z., Sousa, S. G., Santos, N. C., et al. 2012, A\&A, 545, A32 Bensby, T., Alves-Brito, A., Oey, M. S., Young, D., \& Melendez, J. 2011, ApJ, 735, L46

Bovy, J., Rix, H.-W., Liu, C., et al. 2012, ApJ, 753, 148

Casagrande, L., Ramírez, I., Meléndez, J., Bessell, M., \& Asplund, M. 2010, A\&A, 512, A54

Cheng, J. Y., Rockosi, C. M., Morrison, H. L., et al. 2012a, ApJ, 746, 149 Cheng, J. Y., Rockosi, C. M., Morrison, H. L., et al. 2012b, ApJ, 752, 51 Chiappini, C., Matteucci, F., \& Gratton, R. 1997, ApJ, 477, 765

Comerón, S., Elmegreen, B. G., Knapen, J. H., et al. 2011, ApJ, 741, 28 Danovich, M., Dekel, A., Hahn, O., Ceverino, D., \& Primack, J. 2015, MNRAS, 449, 2087

Di Matteo, P., Haywood, M., Gómez, A., et al. 2014, A\&A, 567, A122

Di Matteo, P., Gomez, A., Haywood, M., et al. 2015, A\&A, 577, A1

Dutton, A. A., \& van den Bosch, F. C. 2009, MNRAS, 396, 141

Feng, Y., \& Krumholz, M. R. 2014, Nature, 513, 523

Ferguson, A. M. N., \& Clarke, C. J. 2001, MNRAS, 325, 781

Fisher, D. B., \& Drory, N. 2010, ApJ, 716, 942 
M. Haywood et al.: Clues to the formation of the Milky Way's thick disk

Fraternali, F. 2014, IAU Symp. 298, eds. S. Feltzing, G. Zhao, N. A. Walton, \& P. A. Whitelock, 228

Fuhrmann, K., Chini, R., Haas, M., et al. 2012, ApJ, 761, 159

Haywood, M. 2008, MNRAS, 388, 1175

Haywood, M. 2014a, Mem. Soc. Astron. It., 85, 253

Haywood, M. 2014b, in Lessons from the local group - A Conference in Honour of David Block and Bruce Elmegreen, eds. K. C. Freeman, B. G. Elmegreen, D. L. Block, \& M. Woolway (New York: Springer), in press

Haywood, M., Di Matteo, P., Lehnert, M. D., Katz, D., \& Gómez, A. 2013, A\&A, 560, A109

Hopkins, P. F., Kereš, D., Oñorbe, J., et al. 2014, MNRAS, 445, 581

Iwamoto, K., Brachwitz, F., Nomoto, K., et al. 1999, ApJS, 125, 439

Jørgensen, B. R., \& Lindegren, L. 2005, A\&A, 436, 127

Karakas, A. I. 2010, MNRAS, 403, 1413

Kormendy, J., Drory, N., Bender, R., \& Cornell, M. E. 2010, ApJ, 723, 54

Kroupa, P. 2001, MNRAS, 322, 231

Kubryk, M., Prantzos, N., \& Athanassoula, E. 2015, A\&A, in press,

DOI: $10.1051 / 0004-6361 / 201424171$

Kunder, A., Koch, A., Rich, R. M., et al. 2012, AJ, 143, 57

Laurikainen, E., Salo, H., Athanassoula, E., Bosma, A., \& Herrera-Endoqui, M. 2014, MNRAS, 444, L80

Lehnert, M. D., Di Matteo, P., Haywood, M., \& Snaith, O. N. 2014, ApJ, 789, L30
Madau, P., \& Dickinson, M. 2014, ARA\&A, 52, 415

Marasco, A., Fraternali, F., \& Binney, J. J. 2012, MNRAS, 419, 1107

Marinacci, F., Fraternali, F., Nipoti, C., et al. 2011, MNRAS, 415, 1534

Minchev, I., Chiappini, C., \& Martig, M. 2013, A\&A, 558, A9

Morishita, T., Ichikawa, T., Noguchi, M., et al. 2015, ApJ, submitted [arXiv: 1502.05713]

Naab, T., \& Ostriker, J. P. 2006, MNRAS, 366, 899

Nelson, E. J., van Dokkum, P. G., Brammer, G., et al. 2012, ApJ, 747, L28

Ness, M., Freeman, K., Athanassoula, E., et al. 2013a, MNRAS, 430, 836

Ness, M., Freeman, K., Athanassoula, E., et al. 2013b, MNRAS, 432, 2092

Nidever, D. L., Bovy, J., Bird, J. C., et al. 2014, ApJ, 796, 38

Nissen, P. E., \& Schuster, W. J. 2010, A\&A, 511, L10

Nomoto, K., Tominaga, N., Umeda, H., Kobayashi, C., \& Maeda, K. 2006, Nucl. Phys. A, 777, 424

Raiteri, C. M., Villata, M., \& Navarro, J. F. 1996, A\&A, 315, 105

Schuster, W. J., Moreno, E., Nissen, P. E., \& Pichardo, B. 2012, A\&A, 538, A21

Shen, J., Rich, R. M., Kormendy, J., et al. 2010, ApJ, 720, L72

Snaith, O. N., Haywood, M., Di Matteo, P., et al. 2014, ApJ, 781, L31

Snaith, O., Haywood, M., Di Matteo, P., et al. 2015, A\&A, 578, A87

van Dokkum, P. G., Leja, J., Nelson, E. J., et al. 2013, ApJ, 771, L35

Vollmer, B., \& Leroy, A. K. 2011, AJ, 141, 24

Woods, R. M., Wadsley, J., Couchman, H. M. P., Stinson, G., \& Shen, S. 2014 MNRAS, 442, 732 